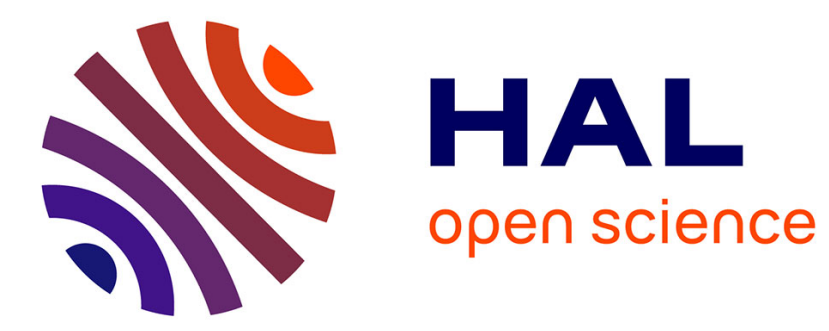

\title{
Tilting preserves finite global dimension
}

Bernhard Keller, Henning Krause

\section{To cite this version:}

Bernhard Keller, Henning Krause. Tilting preserves finite global dimension. Comptes Rendus. Mathématique, 2020, 358 (5), pp.563 - 570. 10.5802/crmath . hal-02968210

\section{HAL Id: hal-02968210 https://hal.sorbonne-universite.fr/hal-02968210}

Submitted on 15 Oct 2020

HAL is a multi-disciplinary open access archive for the deposit and dissemination of scientific research documents, whether they are published or not. The documents may come from teaching and research institutions in France or abroad, or from public or private research centers.
L'archive ouverte pluridisciplinaire HAL, est destinée au dépôt et à la diffusion de documents scientifiques de niveau recherche, publiés ou non, émanant des établissements d'enseignement et de recherche français ou étrangers, des laboratoires publics ou privés. 


\section{Comptes Rendus Mathématique}

Bernhard Keller and Henning Krause

\section{Tilting preserves finite global dimension}

Volume 358, issue 5 (2020), p. 563-570.

<https://doi.org/10.5802/crmath.72>

(C) Académie des sciences, Paris and the authors, 2020.

Some rights reserved.

(G.) Er This article is licensed under the

Creative Commons Attribution 4.0 International License.

http://creativecommons.org/licenses/by/4.0/

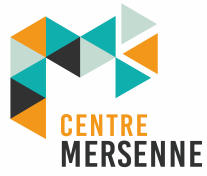

Les Comptes Rendus. Mathématique sont membres $d u$ Centre Mersenne pour l'édition scientifique ouverte www.centre-mersenne.org 


\title{
Tilting preserves finite global dimension
}

\author{
Bernhard Keller*, $a$ and Henning Krause ${ }^{b}$ \\ ${ }^{a}$ Université de Paris, UFR de Mathématiques, Institut de Mathématiques de \\ Jussieu-PRG, UMR 7586 du CNRS, Case 7012, Bâtiment Sophie Germain, 75205 Paris \\ Cedex 13, France \\ ${ }^{b}$ Fakultät für Mathematik, Universität Bielefeld, 33501 Bielefeld, Germany. \\ E-mails: bernhard.keller@imj-prg.fr,hkrause@math.uni-bielefeld.de.
}

\begin{abstract}
Given a tilting object of the derived category of an abelian category of finite global dimension, we give (under suitable finiteness conditions) a bound for the global dimension of its endomorphism ring. 2020 Mathematics Subject Classification. 18G80, 18G20.
\end{abstract}

Manuscript received 2nd February 2020, revised and accepted 11th May 2020.

\section{Introduction}

Tilting theory [1] allows us to construct derived equivalences in various settings. Prime examples are the derived equivalences between algebras obtained from tilting modules [9] or tilting complexes [21] and the derived equivalences between algebras and (non commutative) varieties obtained from tilting bundles, cf. for example $[2,3,8,11]$. An important consequence of the existence of a derived equivalence is the agreement of various subordinate invariants. For instance, the Grothendieck group [21] and Hochschild cohomology [10, 15, 22] are preserved. Another invariant is the finiteness of global dimension, to which this note is devoted. It is well-known that finiteness of global dimension is preserved when two algebras are linked by a tilting module [9, III.3.4] or a tilting complex $[7,12.5]$. Similar facts hold in the geometric examples. It seems natural to unify the algebraic and geometric examples by considering the following general question:

Question. Given a tilting object $T$ in the (bounded) derived category of an abelian category $\mathscr{A}$, does finite global dimension of $\mathscr{A}$ imply finite global dimension of the endomorphism ring of $T$ ?

Despite the ubiquity of tilting objects in algebra and geometry, there seems to be no general result in the literature which guarantees that tilting preserves finite global dimension, even when the category $\mathscr{A}$ is hereditary. ${ }^{1}$ An explanation may be possible confusion about the very definition

\footnotetext{
${ }^{*}$ Corresponding author.

${ }^{1}[19$, Theorem 6.1] claims that $\operatorname{End}(T)$ has finite global dimension when $\mathscr{A}$ is hereditary, but the proof seems to be incomplete.
} 
of a tilting object. In fact, there are various possible definitions in the literature, and we need to clarify this point.

Let $\mathscr{A}$ be an abelian category. By definition, its global dimension is the infimum of the integers $d$ such that $\operatorname{Ext}_{\mathscr{A}}^{i}(-,-)=0$ for all $i>d$. Denote by $\mathbf{D}(\mathscr{A})$ the derived category of $\mathscr{A}$. Fix an object $T \in \mathbf{D}(\mathscr{A})$ and set $\Lambda=\operatorname{End}(T)$. We assume that $\operatorname{Hom}\left(T, \Sigma^{i} T\right)=0$ for all $i \neq 0$.

We consider two settings for $T$ to be a tilting object, depending on whether the abelian category $\mathscr{A}$ is essentially small or not. For the first setting, we focus on the bounded derived category $\mathbf{D}^{b}(\mathscr{A})$ of objects with cohomology concentrated in finitely many degrees. Then we define $T \in \mathbf{D}^{b}(\mathscr{A})$ to be tilting if $\mathbf{D}^{b}(\mathscr{A})$ equals the thick subcategory generated by $T .^{2}$ For example, if $\Gamma$ is a right coherent ring of finite global dimension and $\mathscr{A}$ the abelian category $\bmod \Gamma$ of finitely presented right $\Gamma$-modules, then the object $T$ of $\mathbf{D}^{b}(\mathscr{A})$ is tilting if and only if it is isomorphic to a tilting complex in the sense of [21].

Theorem 1. Let $T \in \mathbf{D}^{b}(\mathscr{A})$ be tilting. Suppose that $\mathscr{A}$ is noetherian, that is, each object in $\mathscr{A}$ is noetherian. Then the global dimension of $\Lambda$ is at most $2 d+t$, where $d$ is the global dimension of $\mathscr{A}$ and the smallest integer such that $H^{i} T=0$ for all $i$ outside an interval of length $t$. Moreover, $\mathbf{R H o m}(T,-)$ induces a triangle equivalence $\mathbf{D}^{b}(\mathscr{A}) \stackrel{\sim}{\rightarrow} \mathbf{D}^{b}(\bmod \Lambda)$ when $\Lambda$ is right coherent.

For our second setting, assume that $\mathscr{A}$ is a Grothendieck category so that $\mathbf{D}(\mathscr{A})$ has arbitrary (set-indexed) coproducts given by coproducts of complexes. Recall that an object $C$ of $\mathbf{D}(\mathscr{A})$ is called compact if the functor $\operatorname{Hom}(C,-)$ commutes with arbitrary coproducts. Each compact object lies in $\mathbf{D}^{b}(\mathscr{A})$, cf. Lemma 7 . Then we define $T \in \mathbf{D}(\mathscr{A})$ to be tilting if it is compact and $\mathbf{D}(\mathscr{A})$ equals the localizing subcategory generated by $T$ (the closure under $\Sigma^{ \pm 1}$, extensions and arbitrary coproducts). For example, if $\mathscr{A}$ is the category $\operatorname{Mod} \Gamma$ of all right modules over a ring $\Gamma$, then the tilting objects in $\mathbf{D}(\mathscr{A})$ are precisely those isomorphic to tilting complexes in the sense of [21].

Theorem 2. Let $T \in \mathbf{D}(\mathscr{A})$ be tilting. Then $\mathbf{R H o m}(T,-)$ induces a triangle equivalence $\mathbf{D}(\mathscr{A}) \stackrel{\sim}{\longrightarrow}$ $\mathbf{D}(\operatorname{Mod} \Lambda)$ and $\operatorname{gl} \cdot \operatorname{dim} \Lambda \leq 2 d+t$, where $d$ and $t$ are defined as in Theorem 1.

We deduce Theorem 1 from Theorem 2. The proof uses t-structures and the strategy is inspired by $[7,12.5]$. For Theorem 2 , we compare the canonical t-structure on $\mathbf{D}(\mathscr{A})$ with the canonical one on $\mathbf{D}(\operatorname{Mod} \Lambda)$; this yields the bound for the global dimension of $\Lambda$. For Theorem 1, we embed $\mathscr{A}$ into a Grothendieck category $\overline{\mathscr{A}}$ and employ the fact that a tilting object $T \in \mathbf{D}^{b}(\mathscr{A})$ identifies with a tilting object in the unbounded derived category $\mathbf{D}(\overline{\mathscr{A}})$.

\section{2. t-structures and finite global dimension}

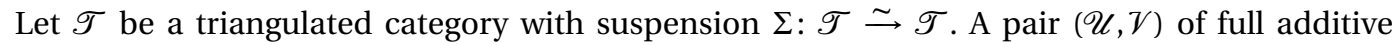
subcategories is called $t$-structure provided the following holds [4]:

(1) $\Sigma \mathscr{U} \subseteq \mathscr{U}$ and $\Sigma^{-1} \mathcal{V} \subseteq \mathcal{V}$.

(2) $\operatorname{Hom}(X, Y)=0$ for all $X \in \mathscr{U}$ and $Y \in \mathcal{V}$.

(3) For each $X \in \mathscr{T}$ there exists an exact triangle $X^{\prime} \rightarrow X \rightarrow X^{\prime \prime} \rightarrow \Sigma X^{\prime}$ such that $X^{\prime} \in \mathscr{U}$ and $X^{\prime \prime} \in \mathcal{V}$.

\footnotetext{
${ }^{2}$ Often the following weaker condition is used: $\operatorname{Hom}\left(T, \Sigma^{i} X\right)=0$ for all $i \in \mathbb{Z}$ implies $X=0$. This is not sufficient in our context.
} 
We consider the following example. Let $\mathscr{A}$ be an abelian category and $\mathscr{T}=\mathbf{D}(\mathscr{A})$ its derived category. For $n \in \mathbb{Z}$ set

$$
\mathscr{T}^{\leq n}:=\left\{X \in \mathscr{T} \mid H^{i} X=0 \text { for all } i>n\right\}
$$

and

$$
\mathscr{T}^{>n}:=\left\{X \in \mathscr{T} \mid H^{i} X=0 \text { for all } i \leq n\right\} .
$$

Then we have $\mathscr{T}^{\leq n}=\Sigma^{-n} \mathscr{T}^{\leq 0}$ and $\mathscr{T}^{>n}=\Sigma^{-n} \mathscr{T}^{>0}$ for all $n \in \mathbb{Z}$. For each $X \in \mathscr{T}$ the truncations in degree $n$ provide an exact triangle

$$
\tau_{\leq n} X \longrightarrow X \longrightarrow \tau_{>n} X \longrightarrow \Sigma\left(\tau_{\leq n} X\right)
$$

with $\tau_{\leq n} X \in \mathscr{T}^{\leq n}$ and $\tau_{>n} X \in \mathscr{T}^{>n}$. Thus the pair $\left(\mathscr{T}^{\leq 0}, \mathscr{T}^{>0}\right)$ is a t-structure and called canonical t-structure on $\mathbf{D}(\mathscr{A})$. Note that the canonical t-structure restricts to the one on $\mathbf{D}^{b}(\mathscr{A})$.

Lemma 3. Let $\left(\mathscr{D}^{\leq 0}, \mathscr{D}^{>0}\right)$ denote the canonical $t$-structure on $\mathbf{D}^{b}(\mathscr{A})$. Then the global dimension of $\mathscr{A}$ is bounded by $d$ if and only if $\operatorname{Hom}(X, Y)=0$ for all $X \in \mathscr{D}^{\geq 0}$ and $Y \in \mathscr{D}^{<-d}$.

Proof. For objects $A, A^{\prime} \in \mathscr{A}$ and $i \in \mathbb{Z}$ we have $\operatorname{Ext}^{i}\left(A, A^{\prime}\right) \cong \operatorname{Hom}\left(A, \Sigma^{i} A^{\prime}\right)$. Thus the global dimension of $\mathscr{A}$ is bounded by $d$ if and only if for all objects $X, Y \in \mathbf{D}^{b}(\mathscr{A})$ with cohomology concentrated in a single degree we have $\operatorname{Hom}(X, Y)=0$ when $X \in \mathscr{D}^{\geq 0}$ and $Y \in \mathscr{D}^{<-d}$. The assertion of the Lemma 3 follows since for $X \in \mathscr{D}^{\geq 0}$ and $Y \in \mathscr{D}^{<-d}$, the truncations induce finite filtrations

$$
X=\tau_{\geq 0} X \rightarrow \tau_{\geq 1} X \rightarrow \tau_{\geq 2} X \rightarrow \cdots
$$

and

$$
\cdots \longmapsto \tau_{<-d-2} Y \longmapsto \tau_{<-d-1} Y \longmapsto \tau_{<-d} Y=Y
$$

such that each subquotient has its cohomology concentrated in a single degree $i$, with $i \geq 0$ for the subquotients of $X$ and $i<-d$ for the subquotients of $Y$.

We wish to extend this Lemma 3 from $\mathbf{D}^{b}(\mathscr{A})$ to $\mathbf{D}(\mathscr{A})$. To this end, we fix a Grothendieck category $\mathscr{A}$. Let us recall some basic facts about derived limits and colimits in $\mathbf{D}(\mathscr{A})$. We will use derived functors in the sense of Deligne [5, 1.2]. Recall that one of the most pleasant properties of Deligne's definition is that for an adjoint pair $(F, G)$, if the derived functors exist, they still form an adjoint pair $(\mathbf{L} F, \mathbf{R} G)$, see for example [14, Section 13]. Let $I$ denote a small category. We write $\mathscr{A}^{I}$ for the Grothendieck category of functors $I \rightarrow \mathscr{A}$. The diagonal functor $\Delta: \mathscr{A}_{\rightarrow} \mathscr{A}^{I}$ taking an object to the constant functor has a left adjoint colim and a right adjoint lim. Let us examine their derived functors. Since the functor $\Delta$ is exact, its left and right derived functors exist and are canonically isomorphic to the induced functor $\mathbf{D}(\mathscr{A}) \rightarrow \mathbf{D}\left(\mathscr{A}^{I}\right)$. For general $I$, the existence of Lcolim is unclear but if $I$ is filtered, then colim is exact (by the definition of a Grothendieck category) and so its left derived functor exists and is canonically isomorphic to the induced functor $\mathbf{D}\left(\mathscr{A}^{I}\right) \rightarrow \mathbf{D}(\mathscr{A})$, which we still denote by colim. This implies in particular that arbitrary coproducts exist in $\mathbf{D}(\mathscr{A})$ and are computed by coproducts in the category of complexes. For arbitrary $I$, the category $\mathscr{A}^{I}$ is still a Grothendieck category. This implies that the right derived functor Rlim exists and is computed as $\operatorname{R} \lim X=\lim \mathbf{i} X$, where $X \rightarrow \mathbf{i} X$ is a homotopy injective resolution in the homotopy category of $\mathscr{A}^{I}$, see for example [12, Theorem 14.3.4]. In particular, this implies that products of arbitrary set-indexed families $\left(X_{i}\right)_{i \in I}$ of objects of $\mathbf{D}(\mathscr{A})$ exist in $\mathbf{D}(\mathscr{A})$ and are computed as products in the category of complexes

$$
\prod_{i \in I} \mathbf{i} X_{i}
$$


where $X_{i} \rightarrow \mathbf{i} X_{i}$ is a homotopy injective resolution for each $i \in I$. For example, if $X_{i}$ is homologically left bounded, then for $\mathbf{i} X_{i}$, we may take any strictly left bounded complex with injective components quasi-isomorphic to $X_{i}$.

Lemma 4. For each complex $X \in \mathbf{D}(\mathscr{A})$ its truncations induce exact triangles

$$
\Sigma^{-1} X \longrightarrow \coprod_{p \geq 0} \tau_{\leq p} X \longrightarrow \coprod_{p \geq 0} \tau_{\leq p} X \longrightarrow X
$$

and

$$
\operatorname{Rim}_{q \leq 0} \tau_{\geq q} X \longrightarrow \prod_{q \leq 0} \tau_{\geq q} X \longrightarrow \prod_{q \leq 0} \tau_{\geq q} X \longrightarrow \Sigma\left(\operatorname{Rlim}_{q \leq 0} \tau_{\geq q} X\right) .
$$

Moreover, we have $X \stackrel{\sim}{\rightarrow} \operatorname{Rlim} \tau_{\geq q} X$ when the injective dimension of each $H^{n} X$ admits a global bound not depending on $n$ and $H^{n} X=0$ for $n \gg 0$.

Remark 5. As explained above, the coproducts in the first triangle may be computed in the category of complexes, whereas the products in the second triangle are products in the derived category or equivalently derived products computed using homotopy injective resolutions. The triangles exist by [16, Proposition A.5 (3)] and its dual but we give a direct proof for the special case at hand below.

Proof. For the first triangle we observe that the colimit of the $\tau_{\leq p} X$ in the category of complexes can be computed degreewise. This gives an exact sequence

$$
0 \longrightarrow \underset{p \geq 0}{\amalg} \tau_{\leq p} X \longrightarrow \coprod_{p \geq 0} \tau_{\leq p} X \longrightarrow X \longrightarrow 0
$$

of complexes and therefore an exact triangle in $\mathbf{D}(\mathscr{A})$, as in the assertion of the Lemma 4 .

For the second triangle we need to construct a K-injective (homotopy injective) resolution of $\left(\tau_{\geq q} X\right)$ in the category of complexes of inverse systems. For each $q<0$, choose an injective resolution $H^{q} X \rightarrow J_{q}$. Then choose a K-injective resolution $\tau_{\geq 0} X \rightarrow I_{0}$ and, for $q<0$, recursively define morphisms $\varepsilon_{q}: I_{q+1} \rightarrow \Sigma^{q+1} J_{q}$ such that we have morphisms of triangles in $\mathbf{D}(\mathscr{A})$

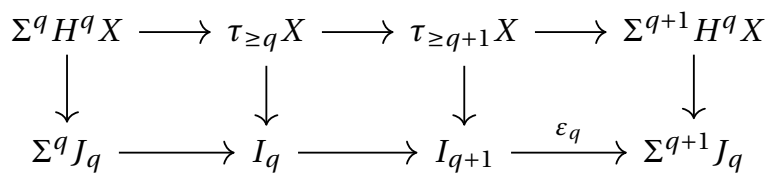

where the vertical morphisms are quasi-isomorphisms and $\Sigma I_{q}$ is the cone over a lift to a morphism of complexes of $\varepsilon_{q}$. The system $\left(I_{q}\right)$ is then quasi-isomorphic to $\left(\tau_{\geq q} X\right)$ and K-injective in the homotopy category of complexes of inverse systems. Thus, it may be used to compute the right derived limit of $\left(\tau_{\geq q} X\right)$. We obtain a degreewise split exact sequence of complexes

$$
0 \longrightarrow \lim I_{q} \longrightarrow \prod_{q \leq 0} I_{q} \longrightarrow \prod_{q \leq 0} I_{q} \longrightarrow 0
$$

and therefore an exact triangle in $\mathbf{D}(\mathscr{A})$, as in the assertion of the Lemma 4 , with

$$
\operatorname{Rlim} \tau_{\geq q} X \cong \operatorname{Rlim} I_{q} \cong \lim I_{q} .
$$

Now suppose that the injective dimension of $H^{q} X$ admits a global bound, say $d$, and we may assume that $H^{q} X=0$ for all $q>0$. To show the isomorphism $X \stackrel{\sim}{\rightarrow} \operatorname{Rlim} \tau_{\geq q} X$ we modify the above construction of a K-injective resolution of $\left(\tau_{\geq q} X\right)$ as follows. For each $q \leq 0$, choose an injective resolution $H^{q} X \rightarrow J_{q}$, where the components of $J_{q}$ vanish in all degrees strictly greater than $d$. We put $I_{0}=J_{0}$ and, for $q<0$, recursively define morphisms $\varepsilon_{q}: I_{q+1} \rightarrow \Sigma^{q+1} J_{q}$ as before. Again, the system $\left(I_{q}\right)$ may be used to compute the right derived limit of $\left(\tau_{\geq q} X\right)$. Since the $J_{q}$ are 
uniformly right bounded, the system $\left(I_{q}\right)$ becomes stationary in each degree. This yields in $\mathbf{D}(\mathscr{A})$ the required isomorphism

$$
X \cong \lim I_{q} \cong \operatorname{Rlim}\left(\tau_{\geq q} X\right) .
$$

Lemma 6. Let $\left(\mathscr{D}^{\leq 0}, \mathscr{D}^{>0}\right)$ denote the canonical $t$-structure on $\mathbf{D}(\mathscr{A})$ and suppose the global dimension of $\mathscr{A}$ is bounded by $d$. Then for $X \in \mathscr{D}^{\geq 0}$ and $Y \in \mathscr{D}^{<-d-2}$ we have $\operatorname{Hom}(X, Y)=0$.

Proof. We apply Lemma 4 . Thus $X$ fits into an exact triangle given by the truncations $\tau_{\leq p} X$, and it suffices to show that $\operatorname{Hom}\left(\tau_{\leq p} X, Y\right)$ and $\operatorname{Hom}\left(\Sigma \tau_{\leq p} X, Y\right)$ vanish for all $p$. On the other hand, $Y$ fits into an exact triangle given by the truncations $\tau_{\geq q} Y$, and therefore it suffices to show that $\operatorname{Hom}\left(\tau_{\leq p} X, \tau_{\geq q} Y\right), \operatorname{Hom}\left(\Sigma \tau_{\leq p} X, \tau_{\geq q} Y\right)$, and $\operatorname{Hom}\left(\Sigma \tau_{\leq p} X, \Sigma^{-1} \tau_{\geq q} Y\right)$ vanish for all $p$ and $q$. This holds by Lemma 3 since both arguments belong to $\mathbf{D}^{b}(\mathscr{A})$.

\section{Tilting for $\mathrm{D}(\mathscr{A})$}

Let $\mathscr{A}$ be a Grothendieck category and $\mathbf{D}(\mathscr{A})$ its unbounded derived category. Recall that the category $\mathbf{D}(\mathscr{A})$ has arbitrary (set-indexed) coproducts given by coproducts in the category of complexes. Notice that the right derived product functor yields arbitrary products in $\mathbf{D}(\mathscr{A})$. In particular, the product of a family of left bounded complexes with injective components is also their product in $\mathbf{D}(\mathscr{A})$.

Lemma 7. If $C$ is a compact object of $\mathbf{D}(\mathscr{A})$, then the cohomology $H^{p} C$ vanishes for all but finitely many integers $p$.

Proof. For each $p \in \mathbb{Z}$, choose a monomorphism $i_{p}: H^{p} C \rightarrow I_{p}$ into an injective object. Using the identification

$$
\operatorname{Hom}_{\mathbf{D}(\mathscr{A})}\left(C, \Sigma^{-p} I\right)=\operatorname{Hom}_{\mathscr{A}}\left(H^{p} C, I\right)
$$

valid for each injective $I$ of $\mathscr{A}$, the $i_{p}$ yield a morphism $i$ from $C$ to the product (in the category of complexes and in the derived category) of the $\Sigma^{-p} I_{p}$. Clearly, in the category of complexes (and hence in the derived category), this product is canonically isomorphic to the corresponding coproduct. So we obtain a morphism from $C$ to the coproduct of the $\Sigma^{-p} I^{p}$ which in cohomology induces the $i_{p}$. By the compactness of $C$, this morphism factors through a finite subcoproduct of the $\Sigma^{-p} I_{p}$ so that all but finitely many of the $i_{p}$ have to vanish. Since they are monomorphisms, the same holds for the $H^{p} C$.

Now let $T$ be a tilting object of $\mathbf{D}(\mathscr{A})$. Thus $T$ is compact, the group $\operatorname{Hom}\left(T, \Sigma^{p} T\right)$ vanishes for all $p \neq 0$, and $\mathbf{D}(\mathscr{A})$ equals its localizing subcategory generated by $T$.

Let $\Lambda$ be the endomorphism ring of $T$. Then $\Lambda$ is quasi-isomorphic to the derived endomorphism algebra $\mathbf{R H o m}(T, T)$ and so the functor $\mathbf{R} \operatorname{Hom}(T,-)$ yields a triangle equivalence

$$
\mathbf{D}(\mathscr{A}) \stackrel{\sim}{\longrightarrow} \mathbf{D}(\operatorname{Mod} \Lambda),
$$

cf. [13]. We use it to identify $\mathbf{D}(\mathscr{A})$ with $\mathbf{D}(\operatorname{Mod} \Lambda)$. The canonical t-structure on $\mathbf{D}(\mathscr{A})$ is denoted by $\left(\mathscr{D}^{\leq 0}, \mathscr{D}^{>0}\right)$, while the canonical t-structure on $\mathbf{D}(\operatorname{Mod} \Lambda)$ is denoted by $\left(\mathscr{D}(\Lambda)^{\leq 0}, \mathscr{D}(\Lambda)^{>0}\right)$.

Lemma 8. Suppose that $\mathscr{A}$ and $\operatorname{Mod} \Lambda$ have finite global dimension. Then the functor $\mathbf{R H o m}(T,-)$ restricts to an equivalence $\mathbf{D}^{b}(\mathscr{A}) \stackrel{\sim}{\rightarrow} \mathbf{D}^{b}(\operatorname{Mod} \Lambda)$.

Proof. Given objects $X, Y \in \mathbf{D}^{b}(\mathscr{A})$ we have $\operatorname{Hom}\left(X, \Sigma^{i} Y\right)=0$ for almost all $i$ since $\mathscr{A}$ has finite global dimension. This is easily shown by induction on the number of integers $n$ such that $H^{n}(X \oplus Y) \neq 0$. It follows that $\mathbf{R H o m}(T,-)$ restricts to a functor $F: \mathbf{D}^{b}(\mathscr{A}) \rightarrow \mathbf{D}^{b}(\operatorname{Mod} \Lambda)$, since

$$
H^{i} \mathbf{R H o m}(T, X) \cong \operatorname{Hom}\left(T, \Sigma^{i} X\right)
$$


and $T \in \mathbf{D}^{b}(\mathscr{A})$ by Lemma 7. On the other hand, $\mathbf{D}^{b}(\operatorname{Mod} \Lambda)$ equals the thick subcategory of $\mathbf{D}(\operatorname{Mod} \Lambda)$ that is generated by the category $\operatorname{Proj} \Lambda$ of projective $\Lambda$-modules, viewed as complexes concentrated in degree zero, since $\Lambda$ has finite global dimension. It follows that $F$ is essentially surjective since $F$ identifies the closure of $T$ under arbitrary coproducts and direct summands with $\operatorname{Proj} \Lambda$.

From now on suppose that the global dimension of $\mathscr{A}$ is bounded by $d$, and fix $t \geq 0$ such that $H^{p} T=0$ for all $p \notin[-t, 0]$, cf. Lemma 7.

Lemma 9. We have $\mathscr{D}(\Lambda)^{\leq 0} \subseteq \mathscr{D}^{\leq 0}$.

Proof. For $X \in \mathscr{D}^{>0}$ and $i \leq 0$ we have $\operatorname{Hom}\left(T, \Sigma^{i} X\right)=0$ since $T \in \mathscr{D}^{\leq 0}$. It follows that $X \in \mathscr{D}(\Lambda)^{>0}$, since $\mathbf{D}(\mathscr{A}) \stackrel{\sim}{\rightarrow} \mathbf{D}(\operatorname{Mod} \Lambda)$ identifies $T$ with $\Lambda$ and $H^{i} X \cong \operatorname{Hom}\left(\Lambda, \Sigma^{i} X\right)$ in $\mathbf{D}(\operatorname{Mod} \Lambda)$. Thus $\mathscr{D}(\Lambda)^{\leq 0} \subseteq \mathscr{D}^{\leq 0}$.

Lemma 10. We have $\mathscr{D}(\Lambda)^{\geq 0} \subseteq \mathscr{D}^{\geq-d-t-2}$.

Proof. Let $X \in \mathscr{D}^{\leq 0}$. Then $H^{i} T=0$ for all $i \notin[-t, 0]$ implies $\operatorname{Hom}\left(T, \Sigma^{i} X\right)=0$ for all $i>d+t+2$ by Lemma 6. It follows that $\mathscr{D}^{\leq 0} \subseteq \mathscr{D}(\Lambda)^{\leq d+t+2}$, and therefore $\mathscr{D}(\Lambda)^{\geq 0} \subseteq \mathscr{D}^{\geq-d-t-2}$.

Proof of Theorem 2. Let $X, Y \in \operatorname{Mod} \Lambda$ and $i>2 d+t+4$. Then

$$
X \in \mathscr{D}(\Lambda)^{\geq 0} \subseteq \mathscr{D}^{\geq-d-t-2} \text { and } \Sigma^{i} Y \in \mathscr{D}(\Lambda)^{<-2 d-t-4} \subseteq \mathscr{D}^{<-2 d-t-4}
$$

by Lemmas 9 and 10. It follows from Lemma 6 that

$$
\operatorname{Ext}^{i}(X, Y)=\operatorname{Hom}\left(X, \Sigma^{i} Y\right)=0 .
$$

Thus the global dimension of $\Lambda$ is bounded by $2 d+t+4$. In order to improve this bound, observe that $\mathbf{R H o m}(T,-)$ restricts to an equivalence $\mathbf{D}^{b}(\mathscr{A}) \stackrel{\sim}{\sim} \mathbf{D}^{b}(\operatorname{Mod} \Lambda)$ by Lemma 8. Then we compare $\mathrm{t}$-structures on $\mathbf{D}^{b}(\mathscr{A})$ and use Lemma 3 instead of Lemma 6 . It follows that the global dimension of $\Lambda$ is bounded by $2 d+t$.

\section{Tilting for $\mathbf{D}^{b}(\mathscr{A})$}

Let $\mathscr{A}$ be an abelian category and $T \in \mathbf{D}^{b}(\mathscr{A})$ a tilting object; recall this means $\operatorname{Hom}\left(T, \Sigma^{i} T\right)=0$ for all $i \neq 0$ and $\mathbf{D}^{b}(\mathscr{A})$ equals the thick subcategory generated by $T$. Set $\Lambda=\operatorname{End}(T)$ and denote by $\operatorname{proj} \Lambda$ the category of finitely generated projective $\Lambda$-modules. By [17, Theorem 3.2], the inclusion add $T \hookrightarrow \mathbf{D}^{b}(\mathscr{A})$ extends to a triangle functor $\mathbf{K}^{b}(\operatorname{add} T) \rightarrow \mathbf{D}^{b}(\mathscr{A})$. Then it is straightforward to show that the composite $\operatorname{proj} \Lambda \stackrel{\sim}{\sim}$ add $T \hookrightarrow \mathbf{D}^{b}(\mathscr{A})$ extends to a triangle equivalence

$$
\mathbf{D}^{b}(\operatorname{proj} \Lambda) \stackrel{\sim}{\longrightarrow} \mathbf{K}^{b}(\operatorname{add} T) \stackrel{\sim}{\longrightarrow} \mathbf{D}^{b}(\mathscr{A}) .
$$

We deduce Theorem 1 from Theorem 2 when $\mathscr{A}$ is noetherian, that is, each object in $\mathscr{A}$ is noetherian. To this end, we fix an essentially small abelian category $\mathscr{A}$ and let $\overline{\mathscr{A}}:=\operatorname{Lex}\left(\mathscr{A}^{\mathrm{op}}, \mathrm{Ab}\right)$ denote the category of left exact functors $\mathscr{A}^{\text {op }} \rightarrow$ Ab. Then $\mathscr{A}$ is a Grothendieck category and the Yoneda embedding $\mathscr{A} \rightarrow \overline{\mathscr{A}}$ which sends $X \in \mathscr{A}$ to $\operatorname{Hom}(-, X)$ is fully faithful and exact, cf. [6, Chapter II].

Lemma 11. Suppose that $\mathscr{A}$ is noetherian and of finite global dimension. Then $\mathbf{D}(\overline{\mathscr{A}})$ is compactly generated (so equals the localizing subcategory generated by all compact objects) and the inclusion $\mathscr{A} \rightarrow \bar{A}$ induces a fully faithful functor $\mathbf{D}^{b}(\mathscr{A}) \rightarrow \mathbf{D}(\overline{\mathscr{A}})$ that identifies $\mathbf{D}^{b}(\mathscr{A})$ with the full subcategory of compact objects. 
Proof. The inclusion $\mathscr{A} \rightarrow \overline{\mathscr{A}}$ identifies $\mathscr{A}$ with the full subcategory of noetherian objects in $\overline{\mathcal{A}}$. It is well-known that an object $I$ of $\overline{\mathscr{A}}$ is injective if and only if $\operatorname{Ext}^{1}(-, I)$ vanishes on all noetherian objects. This implies that the global dimension of $\overline{\mathscr{A}}$ equals that of $\mathscr{A}$.

Let Inj $\overline{\mathscr{A}}$ denote the full subcategory of injective objects and $\mathbf{K}(\operatorname{Inj} \overline{\mathscr{A}})$ the category of complexes up to homotopy. Then the canonical functor $\mathbf{K}(\operatorname{Inj} \overline{\mathscr{A}}) \rightarrow \mathbf{D}(\overline{\mathscr{A}})$ is an equivalence, cf. [18, Proposition 3.6]. It follows that $\mathbf{D}(\overline{\mathscr{A}})$ is compactly generated and that $\mathbf{D}^{b}(\mathscr{A})$ identifies with the full subcategory of compact objects, cf. [18, Proposition 2.3].

Proof of Theorem 1. We apply Lemma 11. The functor $\mathbf{D}^{b}(\mathscr{A}) \rightarrow \mathbf{D}(\overline{\mathscr{A}})$ identifies a tilting object $T$ of $\mathbf{D}^{b}(\mathscr{A})$ with a tilting object of $\mathbf{D}(\overline{\mathscr{A}})$. Let $\Lambda=\operatorname{End}(T)$. Then Theorem 2 provides the bound for the global dimension of $\Lambda$. When $\Lambda$ is right coherent, then the triangle equivalence $\mathbf{D}(\bar{A})$ $\stackrel{\sim}{D}(\operatorname{Mod} \Lambda)$ restricts to an equivalence

$$
\mathbf{D}^{b}(\mathscr{A}) \stackrel{\sim}{\rightarrow} \mathbf{D}^{b}(\operatorname{proj} \Lambda) \stackrel{\sim}{\rightarrow} \mathbf{D}^{b}(\bmod \Lambda)
$$

on the full subcategory of compact objects.

\section{Concluding remarks}

We end this paper with some remarks. Let us fix an essentially small abelian category $\mathscr{A}$ with a tilting object $T \in \mathbf{D}^{b}(\mathscr{A})$, and set $\Lambda=\operatorname{End}(T)$.

Recall that a $\Lambda$-module $X$ is pseudo-coherent if it admits a projective resolution

$$
\cdots \longrightarrow P_{1} \longrightarrow P_{0} \longrightarrow X \longrightarrow 0
$$

such that each $P_{i}$ is finitely generated. We denote by $\operatorname{pcoh} \Lambda$ the full subcategory of pseudocoherent $\Lambda$-modules; it is a thick subcategory of the category of all $\Lambda$-modules, so closed under direct summands, extensions, kernels of epis, and cokernels of monos.

Remark 12. Suppose that $\mathscr{A}$ is noetherian and of finite global dimension. Then $\operatorname{RHom}(T,-)$ induces a triangle equivalence $\mathbf{D}^{b}(\mathscr{A}) \stackrel{\sim}{\longrightarrow} \mathbf{D}^{b}(\operatorname{pcoh} \Lambda)$.

For each pair of objects $X, X^{\prime} \in \mathscr{A}$ we have $\operatorname{Ext}^{i}\left(X, X^{\prime}\right)=0$ for $i \gg 0$. This provides some restriction on the global dimension of $\mathscr{A}$.

Remark 13. Let $\mathscr{A}$ be a length category; thus each object has finite composition length. Then

$$
\operatorname{gl} \operatorname{dim} \mathscr{A}=\inf _{\substack{S, S^{\prime} \\ \text { simple }}}\left\{i \in \mathbb{N} \mid \operatorname{Ext}^{i+1}\left(S, S^{\prime}\right)=0\right\}<\infty
$$

since the number of isoclasses of simple objects is bounded by the length of $H^{*} T$.

Remark 14. The global dimension of $\mathscr{A}$ need not be finite when $\mathbf{D}^{b}(\mathscr{A})$ admits a tilting object. Let $\Lambda$ be a right noetherian ring and set $\mathscr{A}=\bmod \Lambda$. Then $\Lambda \in \mathbf{D}^{b}(\mathscr{A})$ is tilting if and only if each object in $\mathscr{A}$ has finite projective dimension. In this case the global dimension of $\mathscr{A}$ equals the (small) finitistic dimension of $\Lambda$, which may be infinite (even when $\Lambda$ is commutative), cf. [20, Appendix, Example 1].

\section{References}

[1] L. Angeleri Hügel, D. Happel, H. Krause, Handbook of tilting theory, London Mathematical Society Lecture Note Series, vol. 332, Cambridge University Press, 2007.

[2] D. Baer, "Tilting sheaves in representation theory of algebras", Manuscr. Math. 60 (1988), no. 3, p. 323-347.

[3] A. A. Beĭlinson, "Coherent sheaves on $\mathbf{P}^{n}$ and problems in linear algebra", Funkts. Anal. Prilozh. 12 (1978), no. 3, p. 68-69. 
[4] A. A. Beĭlinson, J. Bernstein, P. Deligne, "Faisceaux pervers", in Analysis and topology on singular spaces, I (Luminy, 1981), Astérisque, vol. 100, Société Mathématique de France, 1982, p. 5-171.

[5] P. Deligne, "Cohomologie Etale. Séminaire de Géométrie Algébrique du Bois-Marie 1963-1964 (SGA 4)" (A. Dold, B. Eckmann, eds.), Lecture Notes in Mathematics, vol. 305, Springer, 1973, Avec la collaboration de J.-F. Boutot, A. Grothendieck, L. Illusie et J.-L. Verdier.

[6] P. Gabriel, "Des catégories abéliennes", Bull. Soc. Math. Fr. 90 (1962), p. 323-448.

[7] P. Gabriel, A. V. Roĭter, Representations of finite-dimensional algebras, Encyclopaedia of Mathematical Sciences, vol. 73, Springer, 1992, With a chapter by B. Keller, 1-177 pages.

[8] W. Geigle, H. Lenzing, "A class of weighted projective curves arising in representation theory of finite-dimensional algebras", in Singularities, representation of algebras, and vector bundles (Lambrecht, 1985), Lecture Notes in Mathematics, vol. 1273, Springer, 1987, p. 265-297.

[9] D. Happel, Triangulated categories in the representation theory of finite-dimensional algebras, London Mathematical Society Lecture Note Series, vol. 119, Cambridge University Press, 1988.

[10] _ , "Hochschild cohomology of finite-dimensional algebras", in Séminaire d'Algèbre Paul Dubreil et Marie-Paul Malliavin, Proceedings, Paris 1987-1988 (39ème Année) (M.-P. Malliavin, ed.), Lecture Notes in Mathematics, vol. 1404, Springer, 1989, p. 108-126.

[11] M. M. Kapranov, "On the derived categories of coherent sheaves on some homogeneous spaces", Invent. Math. 92 (1988), no. 3, p. 479-508.

[12] M. Kashiwara, P. Schapira, Categories and sheaves, Grundlehren der Mathematischen Wissenschaften, vol. 332, Springer, 2006.

[13] B. Keller, “Deriving DG categories”, Ann. Sci. Éc. Norm. Supér. 27 (1994), no. 1, p. 63-102.

[14] _ - "Derived categories and their uses", in Handbook of algebra. Vol. 1, vol. 1, North-Holland, 1996, p. 671-701.

[15] _ - "Hochschild cohomology and derived Picard groups”, J. Pure Appl. Algebra 190 (2004), no. 1-3, p. $177-196$.

[16] B. Keller, P. Nicolás, "Weight structures and simple dg modules for positive dg algebras", Int. Math. Res. Not. 2013 (2013), no. 5, p. 1028-1078.

[17] B. Keller, D. Vossieck, “Sous les catégories dérivées”, C. R. Math. Acad. Sci. Paris 305 (1987), no. 6, p. 225-228.

[18] H. Krause, “The stable derived category of a Noetherian scheme”, Compos. Math. 141 (2005), no. 5, p. 1128-1162.

[19] H. Lenzing, "Hereditary categories", in Handbook of tilting theory (L. A. Hügel, ed.), London Mathematical Society Lecture Note Series, vol. 332, Cambridge University Press, 2007, p. 105-146.

[20] M. Nagata, Local rings, Interscience Tracts in Pure and Applied Mathematics, vol. 13, Interscience Publishers; John Wiley \& Sons, 1962.

[21] J. Rickard, “Morita theory for derived categories", J. Lond. Math. Soc. 39 (1989), no. 3, p. 436-456.

[22] _ , "Derived equivalences as derived functors", J. Lond. Math. Soc. 43 (1991), no. 1, p. 37-48. 